\title{
Enfrentar las críticas
}

\author{
Facing $U p^{*}$
}

Alon Harel ${ }^{\star \star}$ y Doron Teichman ${ }^{* \star *}$

Recepción: 30/04/2019

Evaluación: 01/05/2019

Recepción y aceptación final: 29/07/2019

\begin{abstract}
Resumen: Esta respuesta a Moreno Cruz, Richter y Malecka aclara y aborda algunas objeciones a nuestro artículo "Análisis conductista del derecho penal y su aplicación: una amistosa revisión crítica”. En primer lugar, no estamos de acuerdo con el hábil intento de Moreno Cruz de demostrar que la tensión entre la teoría del derecho penal tradicional y el análisis económico-conductista del derecho es un problema falso. Los dos descansan en dos visiones de la naturaleza humana que están en tensión entre sí. Richter plantea la posibilidad de que usar los sesgos para aumentar la disuasión puede ser irónicamente contraproducente, ya que puede erosionar la convicción de que el derecho penal es moral. Proporcionamos razones por las cuales esta hipótesis, aunque es posible, es poco probable que sea cierta. Finalmente, damos la bienvenida a las observaciones de Malecka para examinar los efectos de la legalidad en los incentivos. Es posible que los incentivos puedan ser diferentes cuando se trata de una norma jurídica que cuando se trata de una norma social o moral. Esta preocupación debe ser examinada empíricamente.
\end{abstract}

Palabras clave: Análisis económico-conductista del derecho, Derecho penal, problema falso, influencia mutua, modelado de normas jurídicas

* Traducción del inglés de Bruno Rusca.

** Phillip and Estelle Mizock Chair in Administrative and Criminal Law, The Hebrew University of Jerusalem. Correo electrónico: alon.harel@mail.huji.ac.il

*** Jacob I. Berman Chair in Law, The Hebrew University of Jerusalem. Correo electrónico: doron.teichman@mail.huji.ac.il 


\begin{abstract}
This response to Moreno Cruz, Richter, and Malecka clarifies and addresses some objections to our article "Análisis conductual del derecho penal y su aplicación: Una amistosa revisión crítica". First, we do not agree with Moreno Cruz's skillful attempt to show that the tension between traditional criminal law theory and behavioral law and economics is a false problem. The two rest on two visions of human nature which are in tension with each other. Richter raises the possibility that using biases to increase deterrence may ironically be counter-productive as it may erode the conviction that criminal law is moral. We provide reasons why this hypothesis although possible is unlikely to be true. Finally, we welcome Malecka's observations to examine the effects of legality as such on incentives. It is possible that incentives may differ between the case in which a norm is a legal and a case in which it is a social or moral norm. This concern should be examined empirically.
\end{abstract}

Keywords: Behavioural Law and Economics, Criminal Law, False Problem, Mutual Influence, Modelling Legal Norms

Estamos agradecidos a los tres comentaristas (Diego Moreno Cruz, Anna Ritcher y Magdalena Malecka) por sus observaciones incisivas. El principal problema que plantean los comentaristas alude tangencialmente a la dicotomía entre dos perspectivas sobre el derecho penal: el punto de vista que lo entiende como una forma de inducir y manipular a los individuos para cumplir con el derecho, y el enfoque del derecho penal basado en la racionalidad y el discurso moral. Nos ocuparemos especialmente, aunque no exclusivamente, de este problema central, y no abordaremos, en cambio, todas las objeciones formuladas por los comentaristas. Tampoco pretendemos que nuestras respuestas sean concluyentes; más bien, esperamos que den lugar a nuevas reflexiones sobre la naturaleza del derecho penal y su rol en la sociedad.

\title{
Diego Moreno Cruz
}

En un comentario admirable, meticuloso y muy reflexivo, Moreno Cruz plantea algunas objeciones importantes a nuestro análisis. Nos interesa 
examinar dos puntos claves que él ha señalado. En primer lugar, nos proponemos analizar la afirmación de que no hay un conflicto serio, o al menos, una tensión entre la perspectiva que conceptualiza al derecho penal como un discurso racional entre el delincuente y la sociedad, o como una herramienta para lograr justicia retributiva, y el enfoque que considera al derecho penal como un instrumento para manipular la conducta de los individuos y promover el bienestar social. La segunda cuestión que exploramos es la afirmación de que "la imposición de sufrimiento debe estar basada, independientemente de que existan o no razones instrumentales, en evitar el sufrimiento futuro, y no en el sufrimiento mismo." Por lo tanto, según este punto de vista, para ser legítimo, el derecho penal debe tener algún efecto disuasorio. Es inconcebible pensar en el derecho penal sin considerar la disuasión.

En una sección desafiante de su comentario, titulada "el falso problema", Moreno Cruz argumenta que la tensión que es central en nuestro artículo entre las dos visiones del derecho penal plantea un falso dilema.

Moreno Cruz piensa que:

"El problema de la inconsistencia normativa teórica planteado por Harel y Teichman se aprovecha de una ambigüedad: no diferencian entre la influencia que ejerce el derecho a través de las reglas penales como razones para la acción, y las herramientas y tácticas que utiliza el Estado para que la gente obedezca las leyes penales. Este problema es deficiente en dos sentidos: el análisis económico conductista del derecho y las teorías deontologistas del derecho penal basadas en la retribución (y en la comunicación racional por medio del juicio) son incomparables porque cada uno de estos enfoques analíticos son de distinta naturaleza, y refieren a diferentes problemas y funciones del derecho penal 2) Y estas funciones no son inconsistentes entre sí, es decir, la justificación instrumental del castigo es coherente con la justificación no instrumental de la imposición del castigo fundada en el solo merecimiento del acusado como un resultado del juicio..."

Y más adelante: 
"Por otro lado, la función comunicativa del proceso penal y la función preventiva del nudging ${ }^{1}$ penal no son mutuamente excluyentes. La funciones del derecho penal de prevención, imposición justificada de penas y ofrecimiento de la oportunidad de una comunicación racional en el juicio penal son complementarias y coherentes para maximizar el libre albedrío y la libertad individual en el marco de un sistema penal coercitivo."

Este es un intento admirable de abordar la tensión entre los tradicionales enfoques moralistas del derecho penal y el punto de vista instrumentalista orientado hacia el futuro del análisis económico conductista del derecho. Sin embargo, creemos que, finalmente, fracasa por dos razones.

A este respecto, es necesaria una aclaración inicial. En nuestra contribución original no argumentamos que existe una contradicción en un sentido fuerte entre la visión moral y la instrumentalista del derecho penal. Naturalmente, un sistema penal puede intentar combinar e integrar los enfoques moralistas y retribucionistas del derecho penal con los puntos de vista instrumentalistas. No obstante, argumentamos que este no es un matrimonio feliz y, de hecho, por varias razones, hay tensiones persistentes entre estas dos visiones del derecho penal.

En primer lugar, al menos tradicionalmente, algunas de las perspectivas retribucionistas pretenden exclusividad en la etapa del juicio penal y la imposición de la pena. Además, esta pretensión de exclusividad no es accidental; es parte de la prominente tradición kantiana, que niega enfáticamente que las consideraciones instrumentales tengan alguna relevancia para el derecho penal.

Pero aún si rechazamos el rígido punto de vista kantiano, todavía persiste una tensión entre las dos visiones del derecho penal, ya que no se trata simplemente de dos enfoques del derecho penal, sino también de dos concepciones de la naturaleza humana. Cuanto más son los seres humanos seres racionales que deliberan y actúan sobre la base de juicios morales, menos pueden ser manipulados y tratados como autómatas y viceversa.

1 [N. del T.: en líneas generales, en el marco del análisis económico conductista, el término nudge hace referencia a modos sutiles e indirectos de influenciar la conducta de los individuos, en contraposición a formas más severas de intervención, como prohibiciones o mandatos. Dado que su traducción literal ("dar un empujoncito") no permite captar dicho significado, se ha preferido mantener el término original en inglés.] 
De ahí que la disposición para apoyar una u otra visión del derecho penal dependa de la disposición para adoptar una determinada concepción de la naturaleza humana. Y, si bien las personas podrían ser tanto seres racionales que comparten el reino de los fines y también seres materiales sujetos a manipulación psicológica, cuánto más son lo primero, menos son los segundo y viceversa.

Quizás Moreno Cruz Cruz cree que las dos visiones diferentes del derecho penal son aplicables a distintas etapas o dimensiones del proceso penal. En el juicio penal y en la etapa del castigo, los individuos son tratados como agentes racionales mientras que, por ejemplo, en las etapas de imposición del derecho [law enforcement] ellos son tratados como autómatas manipulables. En cierta medida, esta división es verdadera, pero permítannos plantear dos problemas. El primero es que el análisis económico conductista del derecho no se circunscribe a la etapa de imposición del derecho [law enforcement]; a menudo tiene como objetivo influir en el comportamiento también en la etapa de diseño de las normas legales. Por ejemplo, en un artículo escrito por uno de nosotros, se argumentó que la decisión de usar reglas o estándares en el derecho penal debía regirse por consideraciones conductistas. ${ }^{2}$

En segundo lugar, como ya se ha sostenido, las dos visiones del derecho penal se apoyan en dos concepciones de la naturaleza humana y es irrazonable creer que las personas pueden cambiar radicalmente su naturaleza a medida que pasan de una etapa del proceso penal a otra. De ahí que no pueda defenderse la división tajante entre las dos etapas del proceso penal.

Concentremos nuestra atención en una cuestión diferente planteada por Moreno Cruz Cruz. En su comentario, Moreno Cruz Cruz argumenta:

"Ya sea que se acepte la afirmación retribucionista (que se conforma con los juicios institucionales de las personas) de que la principal función del derecho penal y de la pena no es prevenir que ocurran delitos, también es cierto que la prevención del delito es una consecuencia involuntaria de la criminalización. Cuando un legislador crea una norma penal que tipifica como un delito cierto tipo de acción, es irrazonable negar que el ejercicio de este poder normativo de criminalización tiene por objeto modificar la

2 Ehud Guttel \& Alon Harel, "Uncertainty Reconsidered: Legal Prediction and Legal Postdiction”, Michigan Law Reviw., 107, 2008, pp. 467, 479-487. 
conducta de las personas para que no se cometan delitos, es decir, que el castigo tiene una función prospectiva de prevenir la conducta delictiva de las personas. Es difícil imaginar que la criminalización por parte de los legisladores de cierto tipo de acción no tenga, normalmente, el objetivo de evitar que las personas produzcan daño a otras personas..."

No estamos de acuerdo. Para apoyar nuestra afirmación, permítannos utilizar un argumento y un ejemplo discutido por uno de nosotros en un artículo anterior. ${ }^{3}$ En The Duty to Criminalize, se argumentó que el Estado tiene el deber de criminalizar ciertas clases de comportamiento, incluso si existen formas alternativas de prevenir los delitos. Además, incluso si la criminalización aumenta en lugar de disminuir el índice de criminalidad, a veces hay razones para criminalizarla.

Con arreglo al argumento que se desarrolla allí, el deber de tipificar como delito las violaciones de derechos no se fundamenta en consideraciones instrumentales, a saber, en la mayor probabilidad de que la criminalización contribuya a una mayor protección de la vida y la libertad. En cambio, en ausencia de prohibiciones penales de violaciones al derecho a la vida y a la libertad, los individuos viven "a merced" de los demás. El deber criminalizar se basa, por lo tanto, en la idea de que la vida y la libertad no deberían dejarse a merced de otros individuos, incluso si es probable que estos otros individuos respeten tales derechos.

Para ilustrar el argumento, comparemos dos Estados diferentes: A, B. En el Estado A, la legislatura protege efectivamente la vida y las libertades de sus ciudadanos. Lo hace sin criminalizar las violaciones de estos derechos. Los individuos no son penalmente responsables por tales violaciones, aunque el Estado hace todo lo posible para prevenirlas (utilizando medios no penales). En el Estado B, la legislatura protege efectivamente los derechos mediante el derecho penal. ¿Cuál Estado es moralmente superior?

Los ciudadanos del Estado A están, por supuesto, moralmente obligados a respetar estos derechos y, además, por regla general, puede que efectivamente respeten tales derechos. Sin embargo, dada la ausencia de prohibiciones penales, los derechos de los ciudadanos están "a merced de cusión en: Alon Harel, Why Law Matters, cap. 5, 2014. 
los demás". La protección de sus derechos está sujeta al riesgo de que los individuos puedan cambiar sus opiniones o preferencias. Por lo tanto, bajo este argumento, el Estado B es superior aunque el grado de protección de los derechos sea idéntico en ambos Estados.

¿No basta con garantizar que los individuos estén adecuadamente protegidos de las violaciones de sus derechos? ¿Por qué debería importar si el Estado me protege de las violaciones del derecho a la vida y a la libertad por medio de la criminalización de tales violaciones, o mediante otros (quizás más eficaces o menos costosos) medios no penales? Para defender la afirmación de que es importante, se argumentó que el deber de criminalizar puede justificarse desde el punto de vista de la libertad, entendida como no dominación. La libertad no solo requiere que mis derechos no sean violados; también requiere que mis derechos sean reconocidos públicamente, es decir, que el respeto de estos derechos no esté sujeto a las inclinaciones o juicios de otros. Aunque es posible que (dados los juicios e inclinaciones existentes de las personas) los individuos en el Estado A estén adecuadamente protegidos, todavía están sujetos a dominación, esto es, al riesgo de un cambio potencial en los juicios o inclinaciones de otros individuos. Este riesgo puede ser poco realista; puede incluso ser menos probable que el riesgo de que en el Estado B las prohibiciones legales cambiaran. Pero el hecho mismo de que una persona en el Estado A pueda violar un derecho impunemente es en sí mismo moralmente pernicioso.

Un ejemplo de la vida real, tomado del debate sobre el aborto en Alemania, podría ayudar a subrayar la relevancia del deber de criminalizar, y el valor de la consolidación constitucional de ese deber. Más específicamente, algunos de los debates en torno a los casos de aborto en Alemania deben ser entendidos como debates sobre el deber de criminalizar independientemente de los efectos disuasorios de la criminalización. ${ }^{4}$

En 1975, el Tribunal Constitucional alemán declaró inconstitucional la ley que permitía el aborto a petición durante el primer trimestre de embarazo, por

4 Para la historia de la controversia sobre el aborto en Alemania, véase Donald P. Kommers, "The Constitutional Law of Abortion in Germany: Should Americans Pay Attention?", 10 J. Contemp. Health L. \& Pol'y 1, 1994; Gerald L. Neuman, "Casey in the Mirror: Abortion, Abuse and the Right to Protection in the United States and Germany", 43 Am. J. Compa. L. 273, 1995. 
violar el artículo 2, apartado 2, de la Ley Fundamental alemana que protege el derecho a la vida. ${ }^{5}$ El Tribunal Constitucional enfatizó que el aborto es un acto de homicidio, el cual la ley está obligada a condenar. Como consecuencia de la reunificación, el Tribunal Constitucional tuvo que volver a tratar el asunto, y en su decisión posterior de 1993, reiteró su compromiso con la opinión de que el aborto es, en efecto, una violación al derecho a la vida, y que el Estado tiene la obligación de proteger la vida, incluida la vida del feto.

Con todo, hay diferencias significativas entre los dos casos. Aunque en su sentencia de 1993 el Tribunal reiteró su posición de que el aborto debía seguir siendo un delito, también declaró que el Estado podría sustituir el castigo penal por "asesoramiento normativo" como forma de cumplir con sus obligaciones de proteger a los fetos. El Tribunal declaró que la asesoría obligatoria debía estar diseñada para proteger la vida, y que las disposiciones de cualquier ley futura tendrían que ser específicamente diseñadas con el propósito de preservar la vida del feto, y convencer a la mujer embarazada de que no se practique un aborto.

Si, como creía el Tribunal, en realidad el aborto es una violación del derecho a la vida: ¿por qué alguien estaría dispuesto a despenalizarlo? ¿Cómo podría el Tribunal Constitucional sustituir el castigo penal por el "asesoramiento normativo" como forma de cumplir con sus obligaciones de proteger a los fetos?

Una posible respuesta es que, aunque el poder legislativo tiene el deber de proteger la vida, no tiene el deber de protegerla mediante la criminalización del homicidio; tal vez podría proteger la vida más eficazmente si recurriera a otros medios, tales como la educación, el asesoramiento, etc. Por lo tanto, la disposición a proteger la vida del feto por medios no penales es precisamente lo que diferencia el caso de 1993 del de 1975. En su decisión anterior, el Tribunal resolvió que existe el deber no solo de prevenir el aborto y salvar vidas, sino también de criminalizar el aborto. En cambio, en su decisión posterior, el Tribunal pareció dispuesto a permitir que el

5 Véase 39 BVerFG 1, 1975. Para una explicación del caso y de su importancia, véase Donald P. Kommers, "Liberty and Community in Constitutional law: The Abortion Cases in Comparative Perspective" 1985 BYU. L. Rev. 371; Felix Lange, "American liberalism and Germany's Rejection of the National Socialist Past-the 1973 Roe v. Wade Decision and the 1973 German Abortion / Case in Historical Perspective" 12 German Law Journal 2033, 2011. 
poder legislativo utilizara los medios más efectivos para reducir el número de abortos y, en consecuencia, permitió que los abortos practicados en el primer trimestre del embarazo permanecieran impunes (straffrei).

La diferencia entre la decisión inicial del Tribunal (en la que insistió en mantener un régimen penal) y la decisión posterior, en la que el Tribunal estuvo dispuesto a renunciar a la amenaza de castigo es central para esta discusión. Es indicativo de una diferencia fundamental entre los que se interesan únicamente por proteger la vida de los fetos del modo más efectivo posible, y los que creen que uno también debería mantener una prohibición penal de matar, incluso cuando existen alternativas no penales efectivas. Según este punto de vista, la decisión del Bundestag de despenalizar el aborto simplemente sobre la base de que tal decisión reduce el número de abortos es injustificable, ya que deja a los fetos "a merced" de sus madres. De acuerdo con el acto legislativo que abolió el delito, la decisión de una mujer embarazada de no abortar se habría fundamentado en sus inclinaciones, y no en un derecho públicamente reconocido del feto.

El debate sobre el aborto en Alemania tiene un significado mucho más profundo del que se suele reconocer. Más específicamente, este debate pone de relieve la marcada diferencia entre la protección de la vida y la protección del derecho a la vida. La vida puede ser protegida en un Estado sin que se proteja el derecho a la vida. Además, la protección del derecho a la vida puede incluso ser perjudicial para la protección de la vida, cuando la criminalización del aborto da lugar a un mayor número de abortos. Según la interpretación que aquí se propone, el caso del aborto puede justificarse por el hecho de que la vida y las libertades fundamentales no solo deben protegerse, sino que las violaciones del derecho a la vida y de otras libertades fundamentales deben criminalizarse. De manera más general, este debate demuestra que la suposición de Moreno Cruz de que la disuasión debe ser parte de lo que constituye el derecho penal no es tan obvia como él tiende a creer.

\section{Anna Ritcher}

En su valioso comentario, Anna Ritcher analiza la misma dicotomía entre las dos visiones del derecho penal. Más específicamente, examina un con- 
flicto potencial entre dos dimensiones del análisis conductista del derecho penal. Por un lado, identifica lo que ella denomina "enfoque de la toma de decisiones", que depende fuertemente de las disposiciones psicológicas de los individuos. El enfoque de la toma de decisiones se basa en un conjunto de sesgos identificados por los psicólogos que pueden predecir el comportamiento humano y ser utilizados para manipularlo con el fin de promover la eficiencia. Por otro lado, Richter identifica el "enfoque intuitivo", que se apoya en las concepciones morales predominantes de los ciudadanos. Según esta perspectiva, "el sistema de justicia penal y la administración del castigo" debe orientarse a las percepciones de los ciudadanos sobre la justicia.

Richter sostiene, con razón, que el análisis económico conductista del derecho necesita integrar ambos enfoques. Después de todo, uno de los hallazgos más importantes de los científicos conductistas es que las personas están más dispuestas a cumplir con normas que consideran valiosas y, en la medida en que exista una discrepancia entre sus concepciones morales y el derecho, dicha discordancia socava su disposición a cumplir con las normas. El desafío planteado por Richter es que el uso de sesgos generales para manipular el comportamiento puede ir en contra de los valores morales de la sociedad y, por lo tanto, puede socavar la eficacia del derecho penal. Irónicamente, pues, son precisamente las consideraciones conductistas las que nos obligan a considerar seriamente las concepciones morales sobre derecho penal, ya que ignorar estas concepciones tienen un efecto perjudicial sobre el acatamiento del derecho.

Estamos de acuerdo con esta información y queremos dar un ejemplo. Como mencionamos en nuestro artículo, una de las observaciones importantes del análisis económico conductista del derecho es que nuestras percepciones están influenciadas por un sesgo de accesibilidad. La gente tiende a sobreestimar las probabilidades de que ocurran eventos destacados e intensos. Por lo tanto, parece que una forma de incrementar la disuasión es manipular las percepciones de los individuos sobre la probabilidad de detección. Sin embargo, tal manipulación puede ser percibida como inmoral. Después de todo, la teoría política liberal se basa en el ideal de la transparencia. Los ideales de justificación pública y rendición de cuentas presuponen que los individuos tienen concepciones precisas sobre el funcionamiento de las instituciones públicas. Podría decirse que manipular las 
creencias sobre la probabilidad de detección es incompatible con el ideal liberal de transparencia. Por lo tanto, contrariamente al comentario final de Moreno Cruz en su contribución, no está claro si es una política prudente mentirle a la gente con respecto a la probabilidad de ser atrapado en el aeropuerto de Bogotá mientras se intenta contrabandear drogas.

Esta es una afirmación radical. En la medida en que los individuos crean que el derecho penal trata sobre la moral, un intento de "manipular" las percepciones para aumentar la disuasión podría considerarse en sí mismo como inmoral. Por lo tanto, para mantener la legitimidad del derecho penal, se debería evitar contaminarlo utilizando consideraciones de eficiencia de cualquier tipo. De ello se infiere que el supuesto de que los individuos crean en general que el derecho penal debe regirse exclusivamente por consideraciones morales nos obliga a excluir la relevancia del enfoque de la toma de decisiones, ya que cualquier aplicación de este enfoque entra en conflicto con la centralidad atribuida a las consideraciones morales en el contexto del derecho penal.

Si bien se trata de una posibilidad interesante, se basa en el supuesto de que la gente cree que el derecho penal debe fundamentarse en consideraciones morales. Nosotros creemos que la mayoría de las personas estarían dispuestas a tolerar la relevancia de las consideraciones no morales, en particular, en el ámbito de la imposición del derecho [law enforcement] y del derecho probatorio. Pero, por supuesto, esto es un asunto de investigación empírica. Para establecer la solidez del enfoque de la toma de decisiones no es necesario negar el efecto señalado por Ritcher, a saber, el hecho de que a la gente le importa la moralidad del derecho penal. En cambio, podemos reconocer que el sistema jurídico debería tener en cuenta esta consideración y, por lo tanto, cuando las personas se oponen a un determinado dispositivo de toma de decisiones por razones morales, puede ser recomendable no utilizar este dispositivo. Pero la relevancia de esta consideración puede depender del contexto y no puede implicar un rechazo completo de todos los dispositivos recomendados por el análisis económico conductista del derecho. Solo implica que debemos equilibrar los efectos antagónicos de cada dispositivo.

En conclusión, creemos que Ritcher identificó una observación importante que, dados ciertos supuestos empíricos, puede debilitar el uso del 
tradicional análisis económico conductista del derecho en el contexto del derecho penal. Su desafío debe ser tomado en serio como una limitación en la aplicación de este enfoque en el contexto del derecho penal. Pero tenemos dudas sobre las bases empíricas de su afirmación. Esto refuerza la conclusión de Richter de que: "también se necesitan estudios empíricos acerca de las influencias mutuas entre el enfoque de la toma de decisiones y el de la intuición, y especialmente sobre la cuestión de si sus efectos disuasorios podrían neutralizarse entre sí."

\section{Magdalena Malecka}

Magdalena Malecka plantea una idea interesante. Aunque no está directamente relacionada con nuestro artículo original, merece un análisis cuidadoso. Malecka se propone separar dos aspectos de las normas jurídicas. Por un lado, las normas jurídicas podrían evaluarse exclusivamente en función de su conformidad con normas morales aceptadas. Una norma jurídica puede influir en el comportamiento en la medida en que su contenido sea idéntico a una norma moral. Sin embargo, cuando se analizan algunos experimentos, es evidente que la legalidad de la norma puede influir en la disposición a cumplir con ella. Con otras palabras, el hecho de que la norma sea entendida o conceptualizada en un lenguaje jurídico puede influir en su cumplimiento independientemente de las convicciones morales preexistentes de cada uno. Por ello, al describir el experimento de Kornhauser y Schotter, Malecka señala que:

"Más específicamente, esto puede significar que el 'factor jurídico' tiene un mayor impacto que el 'factor económico' o que el 'factor racional'. Esto sería una prueba de que el aspecto normativo 'jurídico' del derecho (es decir, el hecho de que el comportamiento sea exigido por el derecho) tiene un impacto único en el comportamiento que requiere aclaración.”

Esta observación, no obstante, puede explicarse de dos maneras. Por un lado, la caracterización misma de una norma como "jurídica” puede influir en las percepciones morales de los individuos. En la medida en que las personas crean en la sabiduría de los legisladores y/o crean que el sistema 
es justo, el mero hecho de que una norma sea jurídica puede influir en sus percepciones sobre la moralidad de la norma. Por otro lado, es posible que los individuos crean que existe una obligación general (prima facie) de obedecer el derecho. De ahí que el hecho de que una norma sea una norma jurídica proporcione razones para cumplirla independientemente de la pregunta de si la norma es moral o no. La singular fuerza motivadora de las normas jurídicas puede explicarse de ambas maneras y sería muy interesante examinar cuál de las dos explicaciones permite comprender mejor este fenómeno.

Queremos dar las gracias a los comentaristas por comprometerse con nuestro trabajo y esperamos con entusiasmo el involucramiento en estos temas en el futuro.

\section{Bibliografía}

Guttel, E. y Harel, A., "Uncertainty Reconsidered: Legal Prediction and Legal Postdiction", Michigan Law Review., 107, 2008.

Harel, A., "The Duty to Criminalize", Law and Philosophy, 34 (1), 2015.

Harel, A., Why Law Matters, 2014.

Kommers, D. P., "Liberty and Community in Constitutional law: The Abortion Cases in Comparative Perspective", BYU. L. Rev., 1985.

Kommers, D. P., "The Constitutional Law of Abortion in Germany: Should Americans Pay Attention?", J. Contemp. Health L. \& Pol'y, 10 (1), 1994. Lange, F., "American liberalism and Germany's Rejection of the National Socialist Past-the 1973 Roe v. Wade Decision and the 1973 German Abortion / Case in Historical Perspective", German Law Journal, 12, 2011.

Neuman, G.L., "Casey in the Mirror: Abortion, Abuse and the Right to Protection in the United States and Germany", Am. J. Compa. L., 43, 1995. 